\title{
Actinomicosis torácica: presentación de un caso
} Thoracic actinomycosis: report of a case

\author{
Dr. Martín E. Brizuela ${ }^{a}$, Dr. Antonio Latella ${ }^{b}$, Dra. Julieta Sancho Cano ${ }^{b}$, Bioq. Mirta Litterio ${ }^{c}$ y \\ Dra. Griselda Berberian ${ }^{a}$
}

\section{RESUMEN}

La actinomicosis es una enfermedad producida por bacterias del género Actinomyces. La forma torácica representa el 30\% de los casos. La evolución es habitualmente crónica, con clínica variable.

Se presenta una niña de 11 años de edad con tumefacción subescapular derecha de un mes de evolución, asociada a fiebre, hiporexia y pérdida de peso. Se diagnosticó actinomicosis torácica producida por Actinomyces meyeri. Recibió tratamiento antibiótico durante un año, con resolución completa.

Aunque es poco frecuente, debe ser tenida en cuenta como diagnóstico diferencial en cuadros de tumoración torácica de larga evolución con compromiso del estado general. Debe informarse al microbiólogo la sospecha diagnóstica por lo difícil de su desarrollo en los medios de cultivo habitual.

Palabras clave: actinomicosis torácica, Actinomyces, niño.

\section{ABSTRACT}

Actinomycosis is a disease produced by bacteria of the genus Actinomyces. The thoracic form represents $30 \%$ of the cases. Signs and symptoms are generally unspecific.

A previously healthy 11 year old girl was admitted with a chest mass of a month of evolution associated with fever, hyporexia and weight loss. Thoracic actinomycosis was diagnosed with the isolation of Actinomyces meyeri in the mass drainage. She received antibiotic treatment for a year with complete resolution. Although rare, it should be taken into account as a differential diagnosis in long evolution thoracic tumor affecting the general condition. The microbiologist must be warned about the suspected diagnosis because it is difficult to find it in routinely used culture media.

Key words: Actinomycosis thoracic, Actinomyces, children.

http:/ /dx.doi.org/10.5546/aap.2015.e345

a. Servicio de Control Epidemiológico e Infectología.

b. Servicio de Clínica Pediátrica.

c. Servicio de Microbiología.

Hospital de Pediatría "Prof. Dr. Juan P. Garrahan",

Ciudad Autónoma de Buenos Aires.

Correspondencia:

Dr. Martín Eduardo Brizuela: martin.brizuela1984@gmail.com.

Financiamiento: Ninguno.

Conflicto de intereses: Ninguno que declarar.

Recibido: 30-4-2015

Aceptado: 10-6-2015

\section{INTRODUCCIÓN}

La actinomicosis es una enfermedad producida por bacterias del género Actinomyces. Son anaerobios facultativos que forman parte de la flora habitual orofaríngea, gastrointestinal y del aparato genital femenino. La patología ocurre por disrupción de la mucosa y puede presentarse en tres formas clínicas: cervicofacial, torácica o abdominopelviana. ${ }^{1}$

Es una patología inusual en la edad pediátrica. ${ }^{1,2}$

\section{CASO CLÍNICO}

Niña de 11 años de edad previamente sana, procedente de la provincia de Buenos Aires, que consultó por una tumoración subescapular derecha de un mes de evolución asociada a hiporexia, fiebre intermitente, sudoración nocturna y pérdida del $15 \%$ de peso en los últimos tres meses. Sin antecedentes de viajes, mascotas o contacto con pacientes con enfermedades infecciosas.

Al momento del examen físico, la niña se encontraba afebril, en regular estado general, adelgazada y crónicamente enferma. En la región subescapular paravertebral derecha, se palpaba una tumoración de $10 \times 5 \mathrm{~cm}$, dolorosa, con signos de flogosis. Se observaba una caries en el molar superior izquierdo e hipoventilación en la base del pulmón derecho.

El análisis de laboratorio de ingreso presentaba 9300/ $\mathrm{mm}^{3}$ glóbulos blancos (neutrófilos: 57\%; linfocitos: $36 \%$ ), hemoglobina de $11,5 \mathrm{~g} / \mathrm{dl}$ y $548000 / \mathrm{mm}^{3}$ plaquetas, eritrosedimentación de $10 \mathrm{~mm}$ y proteína $C$ reactiva de $4 \mathrm{~g} / \mathrm{dl}$. Se realizaron hemocultivos, que fueron negativos en aerobiosis y anaerobiosis.

En la radiografía de tórax, se observaba infiltrado alveolointersticial paracardíaco derecho y compromiso costal (Figura 1).

La ecografía mostraba condensación en la base pulmonar derecha con engrosamiento pleural y rarefacción de la cortical de los últimos arcos costales posteriores derechos e imagen fusiforme heterogénea en las partes blandas adyacentes con predominio hipoecoico de $7 \times 8 \times 2 \mathrm{~cm}$ con vasos en su interior. 
La tomografía de tórax con contraste presentaba consolidación pulmonar en el segmento anterior del lóbulo inferior derecho y consolidación basal posterior homolateral. Los músculos paravertebrales derechos se observaron con colección hipodensa, con algunos tabiques de $10 \times 6 \times 3 \mathrm{~cm}$. Los arcos costales posteriores derechos décimo y undécimo, con engrosamiento perióstico sin lesiones óseas (Figura 2).

Sobre la base del cuadro clínico, los diagnósticos diferenciales fueron infección piógena de la piel y las partes blandas con compromiso de estructuras adyacentes: actinomicosis torácica, tuberculosis fistulizada a partes blandas, o bien una infección oportunista, como la aspergilosis pulmonar en un paciente inmunocomprometido. Se inició un tratamiento con ceftriaxona y clindamicina con la punciónaspiración previa de la colección.

Se descartó inmunodeficiencia primaria, inmunodeficiencia adquirida (método ELISA para VIH no reactivo) y tuberculosis (prueba PPD y esputos para bacilos ácido-alcohol resistentes negativos).

FIGURA 1. Radiografía de tórax anteroposterior al momento del ingreso

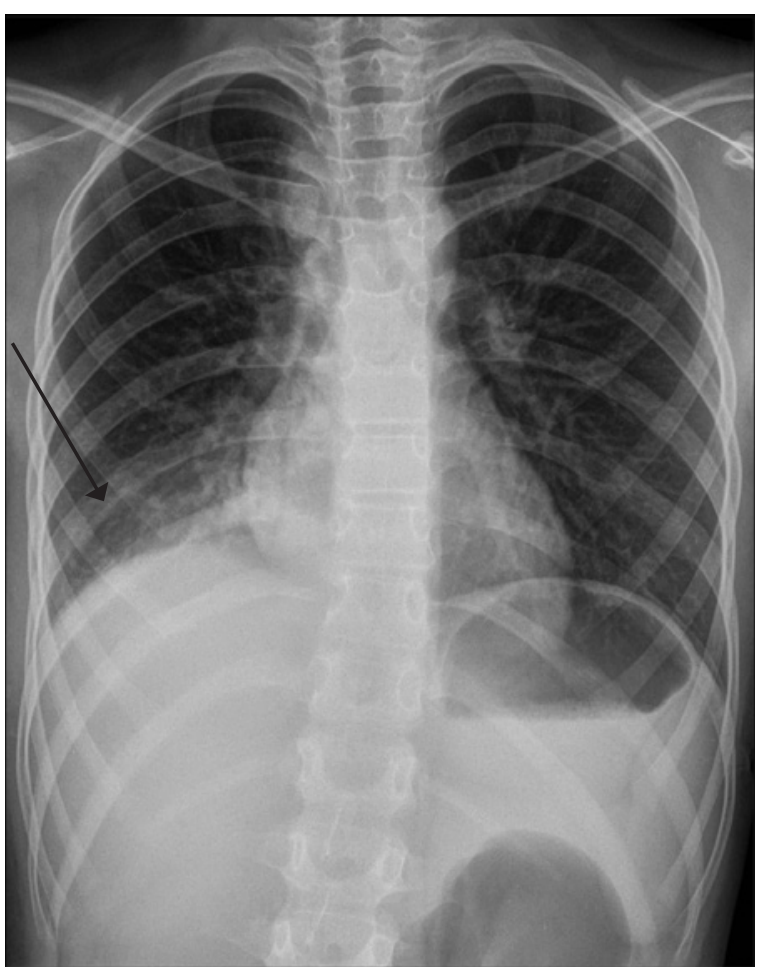

En el examen directo del material purulento, no se observaron gérmenes. Al quinto día de incubación, se obtuvo desarrollo de bacilos Gram positivos difteroides de aspecto irregular compatibles con Actinomyces y, a los doce días de cultivo, se logró la tipificación de Actinomyces meyeri.

La paciente realizó cuatro semanas de tratamiento antibiótico parenteral con penicilina G sódica y completó un total 12 meses con amoxicilina oral. Realizó un tratamiento odontológico de las caries. La evolución clínica fue favorable con resolución de lesiones radiográficas a los dos meses del tratamiento (Figura 3).

\section{DISCUSIÓN}

Las bacterias del género Actinomyces son bacilos Gram positivos, anaerobios facultativos, inmóviles, no formadores de esporas.

Fueron aisladas por primera vez en 1878, en Israel, en muestras de necropsia y el primer caso de actinomicosis torácica fue descrito en 1882 por Ponflick. $^{2}$

Existen alrededor de 21 especies que afectan al ser humano; la más frecuente es Actinomyces israelii. $^{3}$

La forma cervicofacial está favorecida por caries, procedimientos odontológicos, traumatismos, infecciones, cirugía. La torácica se asocia a episodios de aspiración de secreciones y es más frecuente en individuos con trastornos neurológicos o alcohólicos. ${ }^{4} \mathrm{La}$ forma pelviana se asocia al uso de dispositivos intrauterinos de más de 2 años de colocación. ${ }^{5}$

FIGURA 2. Tomografía axial de tórax con contraste endovenoso a la semana de haber ingresado

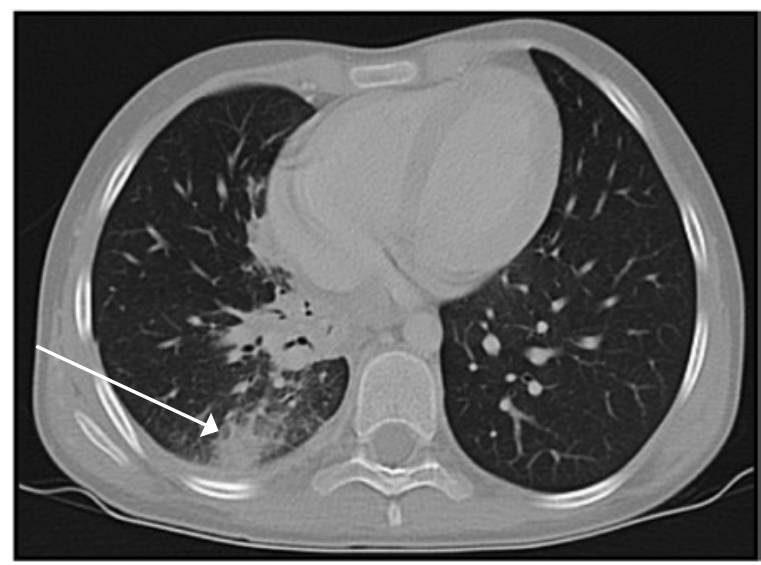


Debido a lo poco frecuente de esta enfermedad, la mayor parte de la bibliografía consiste en reportes de casos.

En el trabajo de Kim, et al. se incluyeron 94 pacientes con actinomicosis pulmonar, todos adultos diagnosticados en Corea entre 2000 y 2010. La neoplasia pulmonar fue el diagnóstico inicial en la mayoría de los casos. ${ }^{3}$

En la revisión de Barlett, et al. en la que se incluyeron 89 casos de actinomicosis pediátrica, la edad más frecuente de presentación fue 10 años, con predominio de sexo masculino. ${ }^{2}$ Mabeza, et al. describen una mayor incidencia entre 11 y 20 años y entre la cuarta y la quinta década de la vida. ${ }^{5}$

Es una patología de presentación subagudacrónica. La clínica varía de acuerdo con la localización: ${ }^{2,3}$

1. Oral o cervicofacial: representa 50\%-65\% de los casos. Existe dolor, trismus, inflamación y drenaje de material purulento en forma de "granos de azufre" por la formación de fístulas cutáneas. Puede producir osteomielitis de mandíbula.

2. Abdominopelviana: representa el $20 \%$ de los casos y afecta, en general, la región ileocecal. Hay fiebre, pérdida de peso y/o masa abdominal. En la forma pelviana, existe sangrado o flujo vaginal, fiebre y pérdida de peso.

Figura 3. Radiografía de tórax de frente a los dos meses de haberse iniciado el tratamiento antibiótico

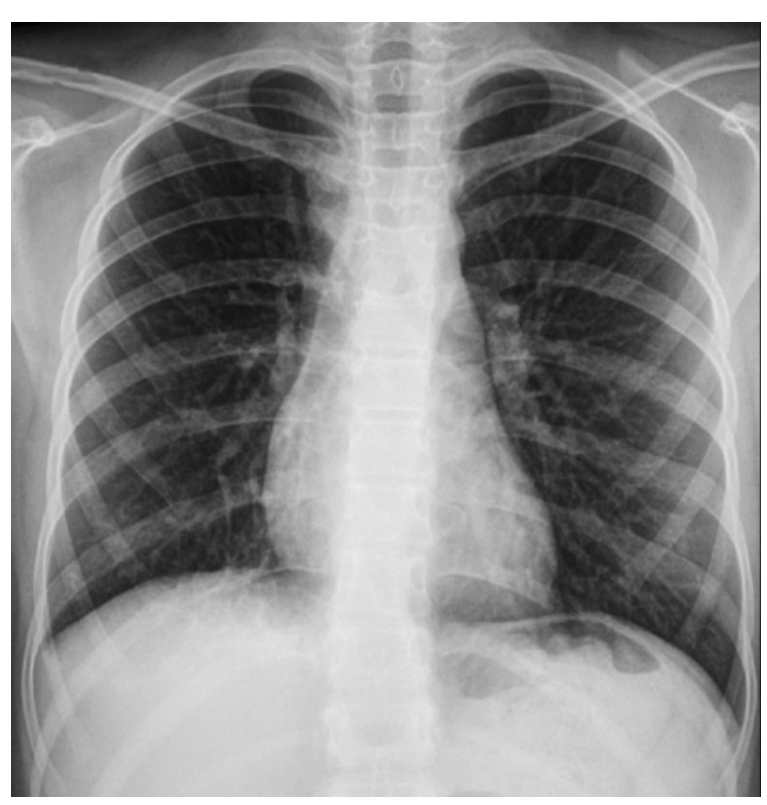

3. Torácica: ocurre en el $15 \%-30 \%$ de los casos. Se presenta con astenia, adinamia, pérdida de peso, con o sin fiebre, dolor o tumefacción de partes blandas torácicas con o sin síntomas respiratorios, como tos productiva y dificultad respiratoria. El compromiso pulmonar, óseo y de partes blandas en un paciente crónicamente enfermo y en buen estado general sumado a la presencia de caries pueden orientar al diagnóstico.

4. Las formas menos frecuentes están representadas por el compromiso neurológico, esquelético, o bien la forma diseminada $(<5 \%)$.

Los hallazgos clínicos más frecuentes de la forma torácica en la infancia son masa torácica $(50 \%)$, tos $(40 \%)$, dolor, fiebre y pérdida de peso (35\% cada uno). ${ }^{2,6}$

Los diagnósticos diferenciales son las neoplasias pulmonares, neumonía bacteriana, micobacterias, aspergilosis y absceso pulmonar, dependiendo de la edad. ${ }^{3,4}$

Son gérmenes fastidiosos que requieren más de tres semanas de incubación para su desarrollo en cultivos habituales y el rédito diagnóstico es menor del $50 \% .^{5}$

En el caso que presentamos, no se observó la presencia de gérmenes en el examen directo; se obtuvo el desarrollo de las bacterias a partir del tercer día de incubación, lo que constituye una debilidad del estudio.

Actinomyces meyeri es una etiología poco frecuente, que fue descrita por primera vez en 1911. En la revisión de Fazili y col., se identifican 32 casos producidos por $A$. meyeri y es el pulmón la localización habitual. El 70\% de los casos se resolvieron con el tratamiento médico. ${ }^{7}$ Comparte la sensibilidad antibiótica con otras especies y tiene predilección por la infección diseminada pero de buen pronóstico. ${ }^{8}$

En el Hospital de Pediatría "Prof. Dr. Juan P. Garrahan", este fue el primer caso de actinomicosis producida por Actinomyces meyeri. Hubo otros dos casos de actinomicosis: uno de localización cervicofacial en un paciente con inmunodeficiencia primaria en el que el agente etiológico fue Actinomyces naeslundii y el tercer caso fue una niña inmunocompetente con la forma torácica, y el diagnóstico fue anatomopatológico sin aislamiento microbiológico.

Histológicamente, las lesiones muestran un centro con contenido purulento, infiltrado neutrofílico y gránulos de azufre rodeados de tejido de granulación con fibras colágenas y fibroblastos. ${ }^{4}$ 
La radiografía de tórax presenta un compromiso periférico y del lóbulo inferior pulmonar. Las lesiones disminuyen a las cuatro semanas de tratamiento adecuado. ${ }^{2}$

En la tomografía de tórax, se observa consolidación en parches, derrame pleural, linfadenopatías regionales y erosión ósea. ${ }^{3,9}$

La consolidación pulmonar con compromiso pleural y de la pared torácica es una tríada característica de actinomicosis torácica. ${ }^{9}$

El tratamiento médico es antibioticoterapia prolongada.

El antibiótico de elección es la penicilina $\mathrm{G}$ sódica. Uniformemente, suele ser sensible a penicilina y amoxicilina. . $^{2,10}$

En pacientes con alergia a la penicilina, pueden indicarse tetraciclinas o eritromicina. Otras opciones son la clindamicina y cefalosporinas de tercera generación. ${ }^{4,7,10}$

Se recomienda el tratamiento parenteral durante 4-6 semanas, seguido de 6 a 12 meses por vía oral. La duración sería mayor en las formas torácicas por la dificultad para alcanzar concentraciones adecuadas de antibiótico dentro del material purulento. ${ }^{4,8}$

La respuesta clínica dentro del mes de haberse iniciado los antibióticos es marcadora de buena evolución a largo plazo. ${ }^{11}$

La cirugía está indicada en casos de necrosis tisular extensa, fístulas, abscesos de gran tamaño, hemoptisis grave, mala evolución con el tratamiento médico y para descartar la etiología neoplásica. ${ }^{12}$ En el caso presentado, no se realizó cirugía por la buena respuesta clínica al tratamiento médico.

\section{CONCLUSIÓN}

Aunque es poco frecuente, la actinomicosis torácica debe considerarse como diagnóstico diferencial en el paciente crónicamente enfermo que se presenta con compromiso pulmonar, óseo y de partes blandas, sumado a la presencia de factores predisponentes, como las caries u otra patología odontológica.

\section{REFERENCIAS}

1. Brook I. Actinomycosis: diagnosis and management. South Med J 2008;101(10):1019-23.

2. Bartlett A, Rivera A, Krishnamurthy R, Baker C. Thoracic actinomycosis in children: case report and review of the literature. Pediatr Infect Dis J 2008;27(2):165-9.

3. Kim SR, Jung LY, Oh IJ, Kim YC, et al. Pulmonary actinomycosis during the first decade of 21st century: cases of 94 patients. BMC Infect Dis 2013;13:216.

4. Pereira N, Cuevas P, Valencia C, Vega J, et al. Actinomicosis torácica como diagnóstico diferencial de neoplasia: a propósito de un caso. Rev Chilena Infectol 2012;29(4):455-8.

5. Mabeza GF, Macfarlane F. Pulmonary actinomycosis. Eur Respir J 2003;21(3):545-51.

6. Morales A, Berardo M, LabaroniC. Actinomicosis torácica, una infección infrecuente en pediatría. Arch Argent Pediatr 2007;105(1):34-7.

7. Fazili T, Blair D, Riddell S, Kiska D,Nagra S. Actinomyces meyeri infection: case report and review of the literature. Infect 2012;65(4):357-61.

8. Choi JCh, Koh WJ, Kim TS, Lee KS, et al. Optimal duration of intravenous and oral antibiotics in the treatment of thoracic actinomycosis. Chest 2005;128(4):2211-7.

9. Heo SH, Shin SS, Kim JW, Lim HS, et al. Imaging of actinomycosis in various organs: a comprehensive review. Radiographics 2014;34(1):19-33.

10. Smith AJ, Hall V, Thakker B, Gemmell CG. Antimicrobial susceptibility testing of Actinomyces species with 12 amtimicrobial agents. J Antimicrob Chemother 2005; 56(2):407-9.

11. Park JY, Lee T, Lee H, Lim HJ, et al. Multivariate analysis of prognostic factors in patients with pulmonary actinomycosis. BMC Infect Dis 2014;14:10.

12. Boudaya MS,SmadhiH,Marghli A, Mouna M, et al.Surgery in thoracic actonomycosis. Asian Cardiovasc Thorac Ann 2012;20(3):314-9. 